See discussions, stats, and author profiles for this publication at: https://www.researchgate.net/publication/338037990

\title{
A Tractable Approximation Approach to Deal with the Binary Nature of Shiftable Loads in Multi-Period Optimal Power Flow
}

Conference Paper · February 2020

DOI: 10.1109/TPEC48276.2020.9042587

\section{CITATIONS}

2

3 authors:

Iason Avramidis

Luxembourg Institute of Science and Technology (LIST)

11 PUBLICATIONS 21 CITATIONS

SEE PROFILE

Geert Deconinck

KU Leuven

475 PUBLICATIONS 5,184 CITATIONS

SEE PROFILE

Some of the authors of this publication are also working on these related projects:

P2P SmarTest Project View project

SNIPPET Secure and Privacy-Friendly Peer-to-Peer Electricity Trading View project
READS

164

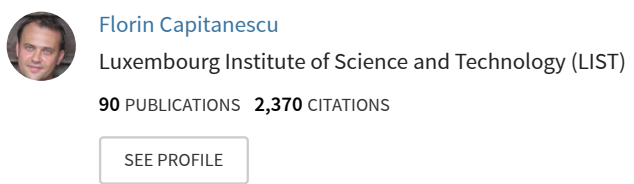




\title{
A Tractable Approximation Approach to Deal with the Binary Nature of Shiftable Loads in Multi-Period Optimal Power Flow
}

\author{
Iason I. Avramidis* ${ }^{\dagger}$, Florin Capitanescu* and Geert Deconinck ${ }^{\dagger}$ \\ *Environmental Research and Innovation, Luxembourg Institute of Science and Technology, Belvaux, Luxembourg \\ ${ }^{\dagger}$ Department of Electrical Engineering, KU Leuven/EnergyVille, Leuven, Belgium \\ Emails: [iason.avramidis, florin.capitanescu]@list.lu, geert.deconinck@kuleuven.be
}

\begin{abstract}
Distribution systems are currently in a transitional phase, as they transform from passive to active. In the coming years, customer participation is expected to drastically increase, as an additional option for maintaining reliable operation of distribution systems and for supporting the distribution and transmission system operators' growing needs. Amongst the available customer-side technologies, the shiftable load (SL) has one of the highest electricity demands and complexity levels; modelling its operation requires a multi-period optimal power flow (MP-OPF) approach. The SL is traditionally modelled in a mixed-integer nonlinear programming (MINLP) setting using many binary variables. However, for larger problem sizes, the MINLP approach lacks tractability. This work proposes a novel NLP approximation of the classic MINLP SL modelling that accurately mimics a SL at the optimum. The proposed approach is validated in a day-ahead deterministic multi-period OPF on two small distribution networks. It is demonstrated that when convergence is achieved, it occurs much faster than for the original formulation. The approach's sensitivity to the chosen level of accuracy and the initialization point are also explored in order to determine the best way to further complement it.

Index Terms-Active Distribution Systems, Demand Response, Multi-Period Optimal Power Flow, Power System Flexibility, Shiftable Load
\end{abstract}

\section{NOMENCLATURE}

\section{A. Sets}

$\mathcal{I} \quad$ Set of buses

$\mathcal{P} \quad$ Set of photovoltaic (PV) panels

$\mathcal{S} \quad$ Set of shiftable loads

$\mathcal{T} \quad$ Set of time periods

$\mathcal{T}_{n w}^{s} \quad$ Set of time periods when SL $s$ must be "OFF"

\section{B. Parameters}

$\begin{array}{ll}A & \text { PV curtailment cost (set to } 25 € / \text { p.u.) } \\ b_{i j} & \text { Susceptance of branch } i-j, \text { p.u. } \\ B & \text { Active power import cost (set to } 10 € / \text { p.u.) } \\ C & \text { SL commitment cost (set to } 5 € / \text { p.u.) } \\ C T_{s} & \text { Cycle time of SL } s, \text { time periods } \\ g_{i j} & \text { Conductance of branch } i-j, \text { p.u. } \\ I_{i j}^{\text {max }} & \text { Line } i-j \text { current limit, p.u. } \\ P_{i, t}^{D} & \text { Active load demand at bus } i, \text { at time period } t, \text { p.u. } \\ P_{p, t}^{g e n} & \text { PV } p \text { generated active power, at time period } t, \text { p.u. }\end{array}$

The authors acknowledge the funding from Luxembourg National Research Fund (FNR) in the framework of gENESiS project (C18/SR/12676686).
$P_{s}^{\text {rate }}$ Rated power of SL $s$, p.u.

$Q_{i, t}^{D} \quad$ Reactive load demand at bus $i$, at time period $t$, p.u.

$S_{i j}^{\max }$ Transformer $i-j$ apparent power limit, p.u.

$T$ Optimization horizon, periods

$t_{s}^{\text {start }}$ Time period $t$ when SL $s$ would normally turn "ON"

$\epsilon^{S L} \quad$ SL approximation factor, \% of SL rated power

\section{Variables}

$P_{i j, t} \quad$ Active power flow at branch $i-j$, at time period $t$, p.u.

$P_{s s, t}^{i m p}$ Imported active power from the MV level, at time period $t$, p.u.

$P_{p, t}^{i n j} \quad$ Active power injected by PV $p$, at time period $t$, p.u.

$Q_{i j, t}$ Reactive power flow at branch $i-j$, at time period $t$, p.u.

$Q_{s s, t}^{i m p}$ Imported reactive power from the MV level, at time period $t$, p.u.

$V_{i, t} \quad$ Voltage magnitude at bus $i$, at time period $t$, p.u.

$\delta_{s, t} \quad$ Binary variable modelling the operation of SL $s$, at time period $t$. It is equal to 1 if the SL is "ON"; otherwise it is equal to 0 .

$\theta_{i, t} \quad$ Voltage angle at bus $i$, at time period $t$, p.u.

\section{INTRODUCTION}

Distribution networks, passive systems until a few years ago, are rapidly transitioning into a new phase of becoming active [1], with the growing deployment of distributed energy resources (renewables and low-carbon technologies). Their large-scale integration continues with a fast pace, creating both opportunities and problems. On one hand, the availability of abundant renewable energy and demand response schemes offers to the distribution system operator (DSO), and by extension to the transmission system operator (TSO), several resources of flexibility, that not only allow for maintaining acceptable operating conditions for the system but also open up many possibilities for bilateral cooperation between DSOs and the TSO [2]. On the other hand, sub-optimal management of any of those resources can lead to network issues, such as voltage and thermal limit violations or unplanned reverse power flows towards higher voltage levels [3].

In the context of demand response (and in trying to avoid renewable production curtailment) the devices with one of 
the highest potentials for operational issues mitigation are shiftable loads (SLs), such as washing machines or dryers. This work focuses on discrete, uninterruptible SLs, although the proposed methodology is applicable to all kinds of SLs. Types of SLs, their mathematical modeling, typical consumption curves and potential value in an electricity market setting are presented in detail in [4]. The accurate modelling of the SLs that we are focusing on is a problematic issue. Devices of that kind have two distinct demand values: zero (SL is "OFF") or their rated power (SL is "ON"). Furthermore, constraints must be enforced to ensure that not only does the modelled device operate for a limited time range (cycle time), and that its operation during that time is continuous (no interruptions).

To the authors' knowledge, most of the works so far that employ SLs introduce binary variables to model SL behavior for each time period within the optimization horizon, leading to various types of mixed-integer problems. The authors of [5] proposed a real-time load control framework, and were among the first to use "auxiliary variables" to model the interchangeability between "ON" and "OFF" states for the examined time periods, in an MILP environment. A similar approach was also employed by the authors of [6] in proposing a demand response scheme with various monetary incentives, which also takes into account periods where the load should remain "OFF". This was expanded by the authors of [7] to a MINLP problem that simultaneously coordinated the actions of a building management system and of an EV aggregator.

The complexity and intractability in large MINLP problems has spawned some research on alternative treatments of SLs, though they are mainly built on an intuitive, rather than a rigorous mathematical basis. The authors of [8] utilize real measurements of SL profiles that actually show slight variations in their consumption levels; SLs do not reach the zero consumption and rated power consumption instantaneously, as there are transitional periods with intermediate consumptions. Thus, considering instead a continuous variable coupled with probability distribution function of actual demand, they propose a heuristic approach to manage the behavior of SLs in a micro-grid setting without employing any mathematical modelling whatsoever. The symbiotic organisms search algorithm is employed by the authors of [9] to optimally schedule the operation of SLs, though this is done for clusters, and thus no proper modelling is employed for individual behaviors. The limited additional literature that deals in some form with SLs either opts to continue using the traditional binary variables approach or employs different heuristic or clustering techniques that eliminate the need for any mathematical modelling.

The work presented in this paper aims to bridge the gap between modelling accuracy of the SL and tractability that is an inevitable concern when traditional modelling would require a prohibitive number of binary variables. By employing a simpler mathematical model that still does not resort to major simplifications a good balance between accuracy and solution speed could be achieved. The major contribution of this paper is a novel NLP approximation of SL modelling, which, to the authors' knowledge, is the first with the potential to achieve the same performance as the traditional MINLP formulation. This work fits the multi-period optimal power flow (MP-OPF) framework, as presented in [10].

The remainder of the paper is structured as follows. In Section III, the standard MINLP mathematical model and the NLP approximation are described. Section IV presents the results from the two test systems and discusses on the findings. Section $\mathrm{V}$ concludes and presents the plans for future work.

\section{PROBLEM DESCRIPTION AND ForMUlation}

\section{A. MP-OPF Basic Setup}

1) Objective function and grid constraints: The examined distribution networks are assumed to be supervised by an "agent" of the DSO. It has a fixed contract in place with each customer, allowing it full control over the customer's devices (PV curtailment or SL shifting), subject to strict technical constraints and predetermined remuneration schemes for activating individual controls. For every time step, its objective is to maintain acceptable voltage magnitude and thermal loading for all nodes and branches respectively, and do so at a minimum cost:

$$
\operatorname{minimize} \sum_{t=1}^{T}\left(E C_{t}^{P V, \text { curt }}+C C^{S L}+E C_{t}^{P^{\text {import }}}\right)
$$

where,

$$
\begin{array}{r}
E C_{t}^{P V, \text { curt }}=\sum_{i \in \mathcal{I}, p \in \mathcal{P}}\left(P_{i, p, t}^{\text {gen }}-P_{i, p, t}^{i n j}\right) \cdot A \\
E C_{t}^{P^{\text {import }}}=P_{t}^{i m p} \cdot B \\
C C^{S L}=\sum_{s \in \mathcal{S}} P_{s}^{\text {rate }}\left(1-\delta_{s, t_{s}^{\text {start }}} \cdot \delta_{s, t_{s}^{\text {start }}+C T_{s}}\right) \cdot C
\end{array}
$$

Eqs. (2)-(3) describe the PV energy curtailment and active power import costs respectively $\left(E C_{t}^{P V, \text { curt }}, E C_{t}^{P^{\text {import }}}\right)$. The SL would normally start and end its operation at $t^{\text {start }}$ and $t^{\text {start }}+C T$ (customer setting). If that is actually the case in the optimum $\left(\delta_{t^{\text {start }}}=\delta_{t^{\text {start }}+C T_{s}}=1\right)$ then the SL was not shifted and the "agent" does not offer any remuneration. If however the optimal solution requires the SL to deviate from that schedule (either or both $\delta_{t^{\text {start }}}, \delta_{t^{\text {start }+C T_{s}}}$ will be zero) then the "agent" pays a fixed commitment cost relative to the SL's size $\left(C C^{S L}\right)$. The above are modelled by Eq. (4).

The MP-OPF problem has to satisfy a number of constraints, detailed hereafter by Eqs. (5)-(12). The PVs' operation is governed by Eq. (5), and are assumed to operate under a unity power factor:

$$
0 \leq P_{p, t}^{i n j} \leq P_{p, t}^{g e n} \quad \forall t \in \mathcal{T}, \forall p \in \mathcal{P}
$$

The technical characteristics of the distribution system in the MP-OPF problem are described by Eqs. (6)-(13), and apply $\forall t \in \mathcal{T}, \forall i, j \in \mathcal{I}: i \neq j$ : 


$$
\begin{array}{r}
P_{i j, t}=-V_{i, t}^{2} g_{i j}+V_{i, t} V_{j, t}\left[g_{i j} \cos \theta_{i j, t}+b_{i j} \sin \theta_{i j, t}\right] \\
Q_{i j, t}=V_{i, t}^{2} b_{i j}+V_{i, t} V_{j, t}\left[g_{i j} \sin \theta_{i j, t}-b_{i j} \cos \theta_{i j, t}\right] \\
\theta_{i j, t}=\theta_{i, t}-\theta_{j, t} \\
\sum_{p \in \mathcal{P}} P_{i, p, t}^{i n j}-\sum P_{i, t}^{D}-\sum_{s \in \mathcal{S}} P_{i, s}^{r a t e} \cdot \delta_{s, t}=\sum P_{i j, t} \\
\sum_{i, t} Q_{i, t}^{D}+\sum_{i \neq j, t} Q_{i j, t}=0 \\
V_{i, t}^{\min } \leq V_{i, t} \leq V_{i, t}^{\max } \\
P_{i j, t}^{2}+Q_{i j, t}^{2} \leq\left(S_{i j, t}^{\text {max }}\right)^{2} \\
P_{i j, t}^{2}+Q_{i j, t}^{2} \leq V_{i, t}^{2}\left(I_{i j, t}^{\text {max }}\right)^{2}
\end{array}
$$

Eqs. (6)-(8) describe the branch power flow equations. Eqs. (9) and (10) represent the nodal power balance constraints. Eqs. (11)-(13) are the voltage magnitude, MV/LV transformer apparent power and line current limits respectively. Note that the active and reactive power flows of the MV/LV transformer are equal to $P_{s s, t}^{i m p}$ and $Q_{s s, t}^{i m p}$ respectively.

2) Conventional SL Modelling: Traditionally, binary variables are used to model discrete SLs. The profile of a SL should have three characteristics [7]: it must have an operational time duration equal to its cycle time, see Eq. (14), its operation must be uninterrupted, see Eq. (15), and it must be "OFF" for specific periods, see Eq. (16):

$$
\begin{array}{r}
\sum_{t=1}^{T} \delta_{s, t}=C T_{s} \quad \forall s \in \mathcal{S} \\
\sum_{t+1}^{t+C T_{s}} \delta_{s, t} \geq C T_{s} \cdot\left(\delta_{s, t+1}-\delta_{s, t}\right) \quad \forall t \in \mathcal{T}, \forall s \in \mathcal{S} \\
\delta_{s, t}=0, \quad \forall t \in \mathcal{T}_{n w}^{s}, \forall s \in \mathcal{S}
\end{array}
$$

The binary nature of $\delta_{s, t}$, coupled with Eqs. (14)-(16), represents the desired behavior of a SL in the frame of a MINLP problem. Thus, the classical MINLP formulation of the MP-OPF problem is described by Eqs. (1)-(16).

However, the high level of detail that is achieved by the MINLP approach is also its biggest weakness; if one wished to simulate a system of $X$ SLs, with a granularity of $Y$ time periods, they would require $X \cdot Y$ binary variables. The computational burden increases manifold and hinders the solution process substantially. An expansion of the problem under the MINLP formulation will suffer degradation in tractability.

\section{B. Proposed NLP Approximation}

To combat the computational burden caused by the binary variables, this paper proposes a novel NLP approximation approach of the original MINLP problem. The proposed approximation is less computationally complex, and additionally guarantees (due to the designed synergy between the new constraints) that when the NLP problem converges, there is a precise imitation (from a technical standpoint) of discrete load behavior by all involved SLs.
The first step is converting $\delta_{s, t}$ to a continuous variable to make the problem NLP. Them, Eq. (15) must be replaced by an equivalent set of constraints. Note that Eq. (14) is maintained in its original form, since it fulfills the SL's fixed energy consumption requirement, which is perhaps the most important constraint. Eq. (16) is also maintained.

The conversion of $\delta_{s, t}$ and the mandate of uninterrupted operation, originally represented by Eq. (16), are equivalently modelled by Eqs. (17)-(19):

$$
\begin{array}{r}
0 \leq \delta_{s, t} \leq 1 \quad \forall t \in \mathcal{T}, \forall s \in \mathcal{S} \\
\sum_{t=1}^{T}\left[\left(\delta_{s, t+1}-\delta_{s, t}\right) \cdot \delta_{s, t+1}\right] \leq 1 \quad \forall s \in \mathcal{S} \\
\sum_{t=1}^{T}\left(\frac{\delta_{s, t}}{\delta_{s, t}+\epsilon^{S L}}\right) \leq C T_{s} \quad \forall s \in \mathcal{S}
\end{array}
$$

Eq. (18) places a tight constraint over the total change in value that $\delta_{s, t}$ can experience. A real uninterruptible SL comes "ON" and goes "OFF" only once over the entire optimization horizon, see Eq. (18). Eq. (19) places a limit on the number of time periods that the SL can be "ON", mimicking an integrality constraint. A real SL can only be "ON" for as long as its cycle time allows, see Eq. (19). $\epsilon^{S L}$ is a small number, used to avoid division by zero. Note that the concept of limiting the number of "ON" periods by using a barrier function shares some similarity with limiting the number of control actions within the scope of an OPF [11]. It was observed to be problematic in achieving convergence, and so the fine-tuning of $\epsilon^{S L}$ is also an important aspect of the proposed approach.

Separately, Eqs. (18) and (19) are insufficient to achieve the desired goal. They are complementary constraints; it is their synergy that achieves the wanted outcome. Notice a major advantage of the approximation approach, as Eq. (18) represents only a single constraint for each SL, contrary to Eq. (15) which actually represents a large set of constraints for all possible values of $t$, thus significantly reducing the computational burden.

It is worth mentioning that ideally, Eqs. (18) and (19) would be strict equalities. However, this would reduce the size of the feasible region significantly and would make achieving a solution harder, especially if the starting point is badly selected. This relaxation is performed to offer additional flexibility to the solver. Besides, it is guaranteed (from a mathematical standpoint) that Eqs. (18) and (19) will be binding at the optimal solution due to their complementarity.

Lastly, it is worth pointing out that while this work focuses exclusively on discrete, uninterruptible SLs, the proposed methodology can easily be extended to interruptible SLs or fixed-time-series profile SLs.

\section{CAse Studies}

\section{A. Description of Test Distribution Systems}

The proposed NLP approach is applied on two low voltage (LV) distribution networks: the 4-bus system (Fig. 1), as a 
proof-of-concept validation, and the 15-bus system (Fig. 2). A realistic $\mathrm{R} / \mathrm{X}$ ratio was used for the lines, following the data presented in [12]. We assume balanced operating conditions and perfect day-ahead forecasting (deterministic approach), which while not a fully realistic simplification for LV feeders, the goal is validating the proposed method's effectiveness from a mathematical perspective before extending it to more specialised settings. Both networks have active/reactive power import capabilities (injected at node 1), installed fixed-demand loads (operating with a 0.9 power factor), installed PVs subject to generation curtailment and discrete, uninterruptible SLs.

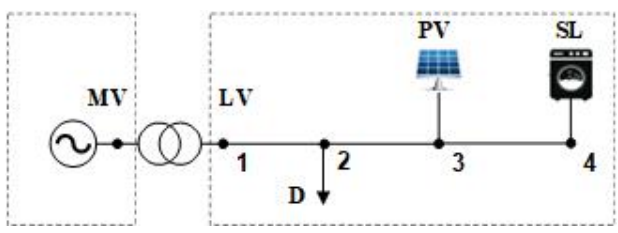

Fig. 1: Layout of 4-bus test system

Realistic load and PV profiles were utilized, created on the basis of real measurements taken from the UK. The load profiles were created using the CREST tool [13]. The original resolution (1-minute intervals) was adapted for time horizons of 6, 12 and 24 periods (hours). The PV profiles were similarly constructed, originally using an update of the CREST tool [14]. A detailed description of the created power profiles can be found in [12]. Base power is $1 \mathrm{~kW}$.

The SLs have different rated powers, ranging between $40 \%$ and $50 \%$ of the maximum single-load active power demand of the system. The SLs' cycle times were set equal to $50 \%$ of the optimization horizon. An abnormally large cycle time was purposefully selected to stress the system. For the 15bus system however, when examining the largest optimization horizon, there were many convergence issues, owing to the (purposefully) problematic setup. Thus, for that case, more realistic cycle times were selected, namely $20 \%$ of the optimization horizon. As was mentioned, optimization horizons of 6, 12 and 24 periods were examined. The MINLP and NLP formulations were solved using the BONMIN solver [15] and IPOPT solver [16] respectively. All simulations were run in the GAMS software, version 28.2 [17].

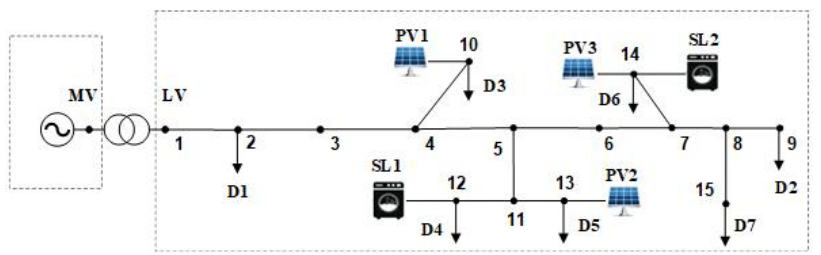

Fig. 2: Layout of 15-bus test system

\section{B. Results using the 4-bus test system}

1) MINLP vs NLP comparison: The comparison between the benchmark MINLP case and the proposed NLP approximation approach are presented in Table I, where a flat start (one for voltage magnitudes, zero for remaining variables), and a value of $\epsilon^{S L}(4 \%)$ that sufficiently represents the SL were selected. The NLP approach is much faster than the MINLP for all three test horizons, achieving computational improvements averaging $78.4 \%$ and reaching $90.5 \%$. It is also very accurate, achieving virtually the same objective value for all optimization horizons (errors less than $0.1 \%$ ). For the current problem size, the NLP approximation is faster than the MINLP and provides a solution of similar quality, despite "launching" the simulation from an unfavorable starting point.

TABLE I: Results, 4-bus system, $\epsilon^{S L}=4 \%$

\begin{tabular}{|c|c|c|c|c|c|c|}
\hline & \multicolumn{2}{|c|}{ Objective } & \multicolumn{2}{c|}{ Solution speed (s) } & \multicolumn{2}{|c|}{ Impr. (\%) } \\
\hline $\mathrm{T}$ & MINLP & NLP & MINLP & NLP & $\Delta f$ & $\Delta t$ \\
\hline 6 & 146.5 & 146.4 & 3.6 & 0.34 & -0.03 & 90.5 \\
12 & 152.4 & 152.3 & 0.43 & 0.12 & -0.04 & 71.6 \\
24 & 159.7 & 159.7 & 3.8 & 1.03 & 0.00 & 73.0 \\
\hline
\end{tabular}

Impr: Improvements, $\Delta f$ : Difference in objective function, $\Delta t$ : Difference in solution time

2) Sensitivity analysis with respect to $\epsilon^{S L}$ : As mentioned previously, the results are expected to be sensitive to the value of $\epsilon^{S L}$. When it is too high then the SL is not adequately modelled. Fig. 3 presents the behavior that the SL exhibits for various values of $\epsilon^{S L}$. When between $10 \%-20 \%$ of the SL's rated power, the observed demand pattern does not properly mimic a real discrete load, as it takes several values between 0 and $P^{\text {rate }}$. When however the value of $\epsilon^{S L}$ decreases sufficiently (here to about $5 \%$ ), the observed behavior of the SL is in line with what one would expect. Do note that the maximum power value of the SL (0.5) p.u. is its rated power (based on the used base power level).

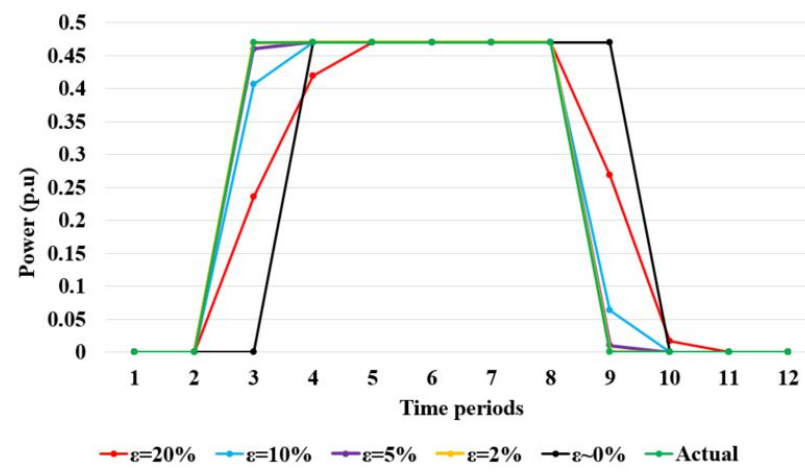

Fig. 3: SL behavior for different $\epsilon^{S L}$ values, 4-bus system, $T=12$

After a certain threshold of $\epsilon^{S L}$, further decrease does not necessarily improve the final solution from an objective value standpoint. Notice that while in Fig. 3 the MINLP (benchmark) behavior and the $\epsilon^{S L} \sim 0$ behavior are dissimilar (different activation and deactivation times for the SL), the objective value is actually the same. This means that the solutions are equivalent, with no preference between them. 
Smaller values of $\epsilon^{S L}$ generally lead to slower solution times. Table II shows that, while not proportionally dependent, increasing the accuracy of the formulation, i.e., tightening the feasible region of Eq. (19), tends to require more iteration by the solver to achieve convergence. This is expected, as $\epsilon^{S L}$ is the main source of numerical instability for the problem. Nonetheless, it was shown that adequate accuracy can be achieved without resorting to too tight constraints. It should be highlighted that even for the slowest case $\left(\epsilon^{S L} \sim 0 \%, 390\right.$ iterations), the NLP formulation achieved a faster solution time (44.22\% improvement) than the MINLP formulation.

TABLE II: Performance of proposed NLP approach for various values of $\epsilon^{S L}$, 4-bus system, $T=24$

\begin{tabular}{|c|c|c|}
\hline$\epsilon^{S L}$ (\% of rated power) & Iterations & Convergence \\
\hline 20 & 94 & $\checkmark$ \\
10 & - & $\times$ \\
8 & 171 & $\checkmark$ \\
7 & - & $\times$ \\
6 & 164 & $\checkmark$ \\
5 & - & $\times$ \\
4 & 274 & $\checkmark$ \\
3 & 184 & $\checkmark$ \\
2 & 139 & $\checkmark$ \\
1 & 240 & $\checkmark$ \\
0.5 & 316 & $\checkmark$ \\
$\sim 0$ & 390 & $\checkmark$ \\
\hline
\end{tabular}

It is as of yet unclear what enables or prohibits convergence to occur. As shown in Table II, the ability to converge certainly depends on the value of $\epsilon^{S L}$. Some values are problematic, while some are not. However, it is not necessarily small values that cause the issues, as even when $\epsilon^{S L}=10 \%$ (a theoretically "safe" numerical point) the solver fails to converge. The convergence behavior depends on the individual feasible regions and starting points. Similar issues were also reported in [11], where an in-house NLP solver was used; it appears that the issues persist even when using a professional NLP solver (IPOPT).

\section{Results using the 15-bus test system}

1) MINLP vs NLP comparison: The network has tripled in size and there are 2 SLs. However, this does not guarantee more observations of non-convergence cases. The 15-bus, $T=12$ case and the 4-bus, $T=24$ case (which has an excellent convergence record) represent an equal number of binary variables (24). As can be seen in Table III (for $T=6, T=12)$, the NLP approach remains very accurate (on average less than $1 \%$ error in the objective function) and significantly faster than the MINLP approach (on average more than $90 \%$ faster).

For the $T=24$ case, more non-convergence cases are observed. 48 binary variables are represented, with the NLP approach now being sensitive to numerical issues. However, when reducing the cycle time from 13 periods to $5(* *)$
TABLE III: Results, 15-bus system, $\epsilon^{S L} \sim 0 \%$

\begin{tabular}{c|c|c|c|c|c|c|}
\hline & \multicolumn{2}{|c|}{ Objective } & \multicolumn{2}{c|}{ Solution speed (s) } & \multicolumn{2}{|c|}{ Impr. (\%) } \\
\hline $\mathrm{T}$ & MINLP & NLP & MINLP & NLP & $\Delta f$ & $\Delta t$ \\
\hline 6 & 105.5 & 106.1 & 11.6 & 1.97 & 0.58 & 83.1 \\
12 & 228.8 & 226.4 & 89.5 & 2.41 & 1.04 & 97.3 \\
$24^{* *}$ & 565.6 & 575.7 & 65.4 & 2.44 & 1.78 & 96.3 \\
$24^{* * *}$ & 463.4 & 463.3 & 33.1 & 0.89 & 0.01 & 97.3 \\
\hline
\end{tabular}

the NLP approach easily converges to an accurate solution 96.3\% faster than the MINLP approach. This shows that the range of the cycle time might actually have a higher impact on the performance than the number of binary variables represented. Furthermore, if we maintain the abnormally large cycle times but provide a sufficient starting point $(* * *)$, i.e., the determination of approximately $60 \%-80 \%$ of the SL's "ON" periods, the NLP approach again converges very fast (97.3\% faster than the MINLP) and with high accuracy (less than $2 \%$ error in the objective function).

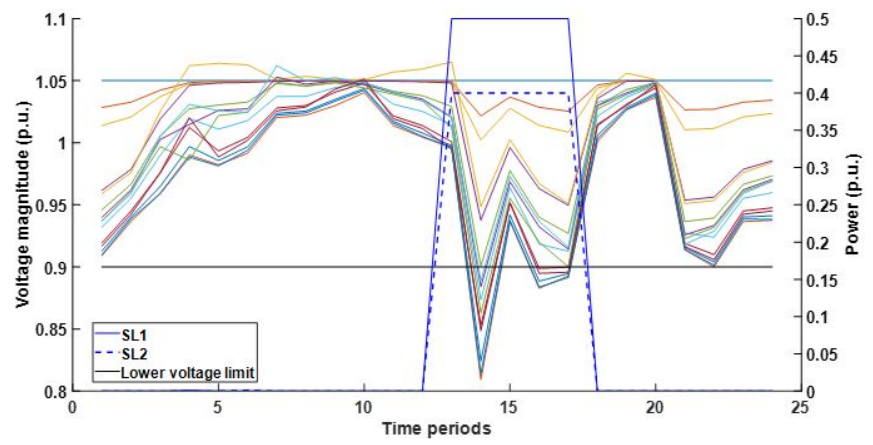

Fig. 4: Voltage behaviors for the 2 unaltered SL profiles, 15bus system, $T=24, C T=5$ (PV curtailment cost: $428 €$ )

2) Impact of the initialization: The impact that the selection of the starting point has on the approach's performance cannot be understated. It is not just achieving convergence that heavily relies on the initialization; the quality of the solution may also be largely affected. Fig. 4 presents the voltage profile of the 15-bus system for the $T=24, C T=5$ case if the SLs are not shifted and maintain their original operation times. As is evident, a large number of undervoltages $\left(V_{i, t}<0.9\right)$ is observed. The PV curtailment cost is $428 €$. Obviously, this situation is undesirable and must be rectified.

With proper initialization (intuition dictates that part of the SL will operate between periods (t) 7 and 11), the NLP approach quickly converges to the same solution as the MINLP. This makes a strong point in favor of combining the NLP approach with proper initialization. Both SLs are shifted to mitigate all voltage issues (Fig. 5), with the PV curtailment cost reduced by more than $10 \%$ ( $382 €$ ), thus simultaneously achieving acceptable operating conditions and cost reduction.

However, when the SLs are badly initialized (in this case 
both between $t=16$ and $t=20$ ) and the voltage limit relaxed, the NLP approach does still converge, but in that case, more undervoltages have actually been created, while not all of the original undervoltages have been eliminated, as is shown in Fig. 6. Additionally, the PV curtailment cost has slightly increased to $433 €$. This is important, because while the PV curtailment is about the same as in the first case, there are more undervoltages. Clearly, the solver has converged a local optimum which is clearly sub-optimal (in comparison to the MINLP case), even though a better solution does exist. The representation of a large number of binary variables using only continuous variables creates a problematic feasible region that if not properly dealt with can easily trap the solver to some local sub-region. This just goes to support the claim that while NLP approach is superior to the MINLP in terms of computation time, it is sensitive to the provided starting point, and thus it must be additionally supported to reliably surpass the MINLP approach in a cosistent basis.

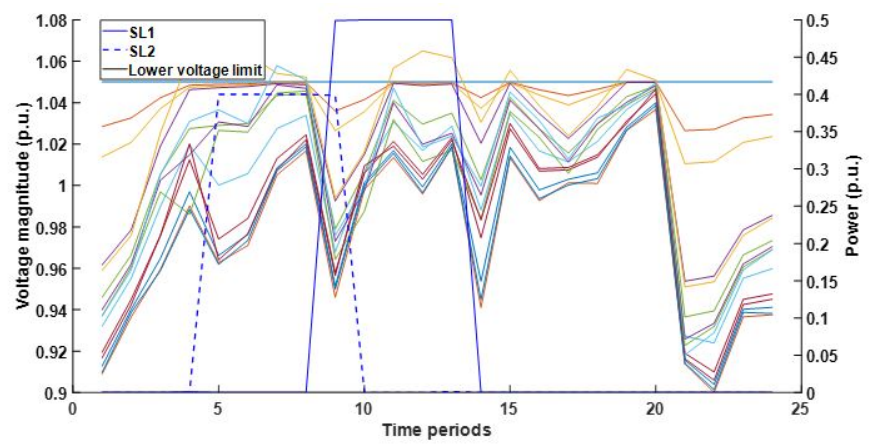

Fig. 5: Voltage behaviors for the 2 optimal SL profiles, 15-bus system, $T=24, C T=5$ (PV curtailment cost: $382 €$ )

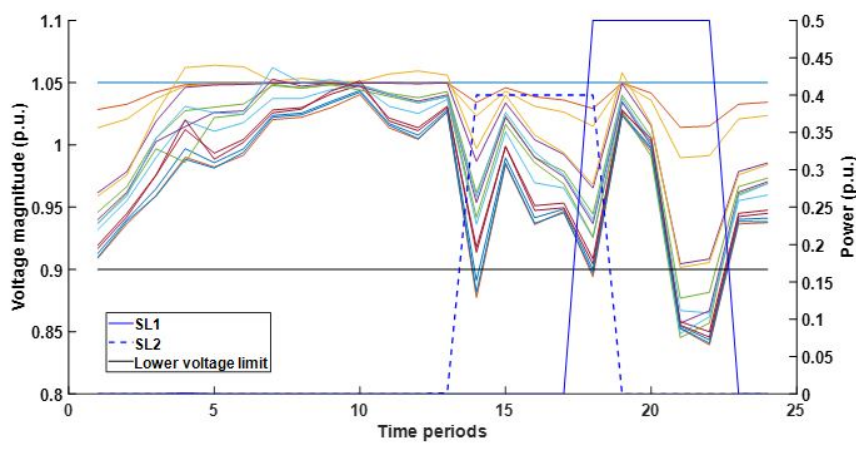

Fig. 6: Voltage behaviors for the 2 sub-optimal SL profiles, 15-bus system, $T=24, C T=5$ (PV curtailment cost: 433 $€)$

\section{CONClusions \& Future Work}

This paper presents a novel NLP approximation to simulate the behavior of shiftable, uninterruptible discrete loads, traditionally modelled using binary variables in a MINLP framework. It is demonstrated that with proper selection of the approximation factor $\epsilon^{S L}$ the proposed approach performs as accurately as the MINLP formulation, at speeds more than $70 \%$ higher. Numerical issues are observed for some value of $\epsilon^{S L}$ or when the number of examined time periods is too high. However, with proper starting point selection, the probability of convergence drastically increase and the NLP problem still outperforms its predecessor by a large margin.

In the near future, the authors plan to validate the proposed approach in larger networks (300 buses), and to devise smart heuristic techniques that would produce good starting points (especially when there are multiple SLs) to further reduce the probability of encountering numerical issues.

\section{REFERENCES}

[1] A. Keane et al. "State-of-the-Art Techniques and Challenges Ahead for Distributed Generation Planning and Optimization”. IEEE Trans. Power Syst., 28(2):1493-1502, May 2013.

[2] G. Migliavacca, M. Rossi, D. Six, M. Dzamarija, S. Horsmanheimo, C. Madina, I. Kockar, and J.M. Morales. "SmartNet: a H2020 project analysing TSO-DSO interaction to enable ancillary services provision from distribution networks". In CIRED, June 2017.

[3] I.I. Avramidis, V.A. Evangelopoulos, P.S. Georgilakis, and N.D. Hatziargyriou. "Demand side flexibility schemes for facilitating the high penetration of residential distributed energy resources". IET Gener Transm. Distr., 12(18):4079-4088, October 2018.

[4] M.C. Vlot, J.D. Knigge, and J.G. HanSlootweg. "Economical Regulation Power Through Load Shifting With Smart Energy Appliances". IEEE Trans. Smart Grid, 4(3):1705-1712, September 2013.

[5] A.H. Mohsenian-Rad and A. Leon-Garcia. "Optimal Residential Load Control With Price Prediction in Real-Time Electricity Pricing Environments". IEEE Trans. Smart Grid, 1(2):120-133, September 2010.

[6] M.R. Sarker, M.A. Ortega-Vazquez, and D.S. Kirschen. "Optimal Coordination and Scheduling of Demand Response via Monetary Incentives". IEEE Trans. Smart Grid, 6(3):1341-1352, May 2015.

[7] J.E. Contreras-Ocana, M.R. Sarker, and M.A. Ortega-Vazquez. "Decentralized Coordination of a Building Manager and an Electric Vehicle Aggregator". IEEE Trans. Smart Grid, 9(4):2625-2637, July 2018.

[8] G. Graditi, M.L. Di Silvestre, R. Gallea, and E.R. Sanseverino. "Heuristic-Based Shiftable Loads Optimal Management in Smart MicroGrids". IEEE Trans. Industrial Inf., 11(1):271-280, February 2015.

[9] R. Cakmak and I.H. Altas. "Optimal Scheduling of Time Shiftable Loads in a Task Scheduling Based Demand Response Program by Symbiotic Organisms Search Algorithm". In SASG, December 2017.

[10] S. Gill, I. Kockar, and G.W. Ault. "Dynamic Optimal Power Flow for Active Distribution Networks". IEEE Trans. Power Syst., 29(1):121131, January 2014.

[11] F. Capitanescu, W. Rosehart, and L. Wehenkel. "Optimal power flow computations with constraints limiting the number of control actions". In IEEE Bucharest PowerTech, June 28-July 2, 2009.

[12] A. Navarro-Espinosa and L.F. Ochoa. "Probabilistic impact assessment of low carbon technologies in LV distribution systems". IEEE Trans. Power Syst., 31(3):2192-2203, July 2015.

[13] I. Richardson, M. Thomson, D. Infield, and C. Clifford. "Domestic electricity use: A high-resolution energy demand model". Energy and Buildings, 42(10): 1878-1887, October 2010.

[14] I. Richardson and M. Thomson. "Integrated simulation of photovoltaic micro-generation and domestic electricity demand: a one-minute resolution open-source model". Journal of Power and Energy, 227(1):73-81, February 2013.

[15] P. Bonami et al. "An Algorithmic Framework for Convex Mixed Integer Nonlinear Programs". Discrete Optimization, 5(2):186-204, May 2008.

[16] A. Wächter and L. T. Biegler. "On the Implementation of a PrimalDual Interior Point Filter Line Search Algorithm for Large-Scale Nonlinear Programming". Mathematical Programming, 106(1):25-57, March 2006.

[17] B.A. McCarl, GAMS user guide, version 23.8, 2012, www.gams.com. 